\title{
Electrical Parameter Detecting System of Jordan Refinner Based on Virtual Instrumentation
}

\author{
Yang Shifeng, FU Peng \\ College of Electronic information and automation \\ Tianjin University of Science \& Technology \\ Tianjin 300222, China \\ yangsf@tust.edu.cn \\ 345333624@qq.com
}

\author{
Yang ye \\ College of Electrical \& Electronic and Computer \\ Science , Florida State University \\ yangye@yahoo.com
}

\begin{abstract}
There are some disadvantages like that singlefunction and lower extent of automation in the electrical parameter detecting system of Jordan refiner, so it's hard to adapt the system to the demand of the large-scale automatic detecting industry. Depending on the jordan refiner used in paper making, basing on the Labwindows/CVI software to be the host computer and the idea of virtual instrumentation, this devise constructed three-phase detecting system of the electrical parameter which using CS5460A power/energy computation chip for AD converter produced by Cirrus Logic company. The hardware construction and the software designing method are introduced; the concrete plan of enhancing the electrical parameter detecting precision is analyzed. The actual test suggests that the whole system is advantaged in high precision, fast speed, shot exploiting period and deeper extent of automation.
\end{abstract}

Keywords- Jordan refiner, electrical Parameter; CS5460A; Labwindows/CVI

\section{INTRODUCTION}

Power measurement devices used in Jordan refiner of papermaking in early time were analog ammeters in majority, which are hard to measure the net power consumption when Jordan refiner is operating. Additionally, this sort of power calculation device has no communication ports with computers, and therefore, power measurement analysis in working process is not achievable. The appearance of the virtual instrumentation [1] and the "CS5460A" energy computation chip changed the traditional concept of the electrical parameter detecting system, which changed the hardware of the traditional instrument into software, merged the software and computer into an integrated system. The new concept took advantage of the resources of software and hardware in computer and integrated the virtual instrument technology with the high precision electrical parameter detecting chip; furthermore, it combined with the computer, which is incomparable with the traditional testing devices in aspects of function, maintenance, application and updating. This system exploited the detecting and analyzing platform with appropriative electrical parameter aimed at papermaking equipments. The current device has been applied to the power measurement system of Jordan refiner successfully which belongs to Beijing Paper Making Graduate School. The whole system is advantaged in simple manipulation, high precision and reliable capability.

\section{DEVISE OF THE HARDWARE}

Presently, there are two designing plans principally in electronic electrical parameter detecting system about the hardware construction. First plan is the ADC DSP method which can revert the basic wave via the FFT transform; second way is that adopting appropriative energy computation chip, CPU can read the parameters and process it simply from interior register of the special energy computation chip. Current system adopted the second plan.

CS5460A is a single-phase energy computation chip [2] with predominant function which the precision is high and the linearity is well. Because of a lot of applications in energy computation, we adopted it to accomplish the mission of the parameter measuring. CS5460A is a single-phase power/energy computation chip, if we use 3 phase- 3 wires, it will needs two CS5460A chips; if we use 3 phase- 4 wires, it will needs three [3]. For the sake of compatibility, three chips method is adopted.

The MCU is ATMEGA162 made in ATMEL company, it is a low-cost, low-power and advanced RISC architecture microcontroller. Moreover, it has abundant interfaces which including 1K RAM, 16K FLASH, so the additional RAM and ROM are not needed to extend.

Fig. 1 shows the actualized hardware frame diagram. The mutual inductor translates the current and voltage into feeble signal less than $150 \mathrm{mv}$, the CS5460A calculates parameters on respective phases according to electrical signals and then transmits the electrical parameters like current power to ATMEGA162 that accomplishes the mission of processing, displaying, storage and transmitting by means of SPI serial interface. As the system clock chip, DS3231 provides the clock information for system; 24C16 is used to save the measured parameters and the checkout value. System receives the low frequency pulse from outside and calibrates the other ammeters, in the mean time, it outputs pulse and calibrated by the other higher precision devices. 


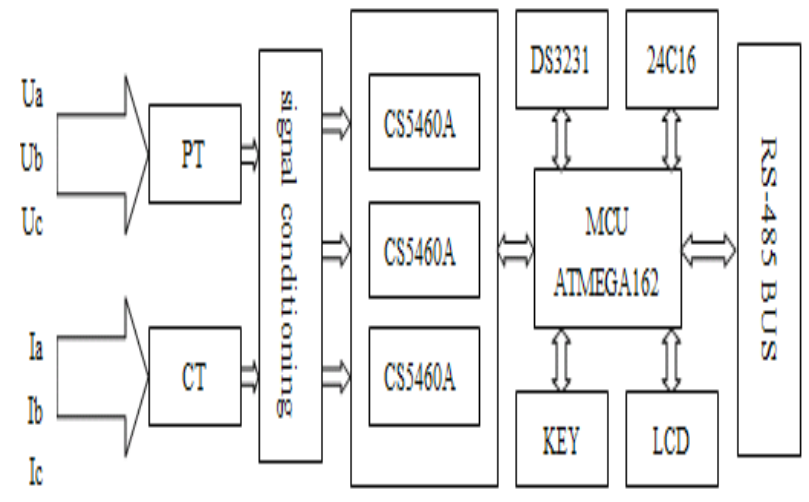

Fig. 1 Hardware implementation frame diagram of system

\section{A. Devise and Actualization of the Hardware Circuit}

The input channel of CS5460A can merely receives weak voltage signal less than $250 \mathrm{mv}$, the strong current and voltage signals from electrical wire need to be adjusted firstly, then it can be connected to the input channel of CS5460A [4]. The voltage channel can adopts the way of resistance potentiometer; the electricity channel can uses the current mutual inductor, so the current and voltage values can be adjusted to the requisite range. The input signal part circuit diagram is as Fig. 2a and Fig. 2b show, R4 is the sampling resistance.

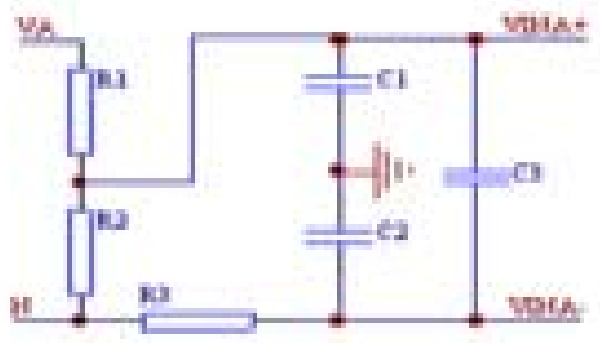

Fig. 2a voltage inputting channel devise

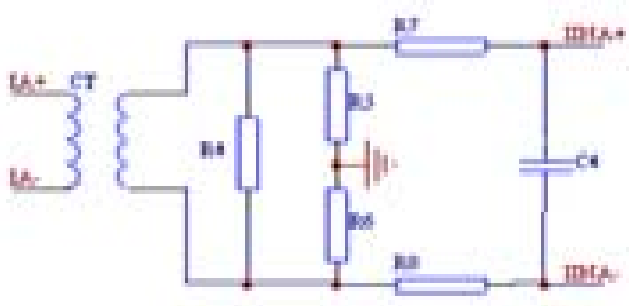

Fig. 2b current inputting channel devise

CS5460A which measuring A-phase electrical parameter is connected as Fig. 3: the other two phases are same as Aphase, 2 pins CPUCLK supplies the benchmark frequency for the next CS5460A, CSA is the chip selecting port, EOUTA is the pulse outputting port which is connected with an exterior interruption pin of ATMEGA162.

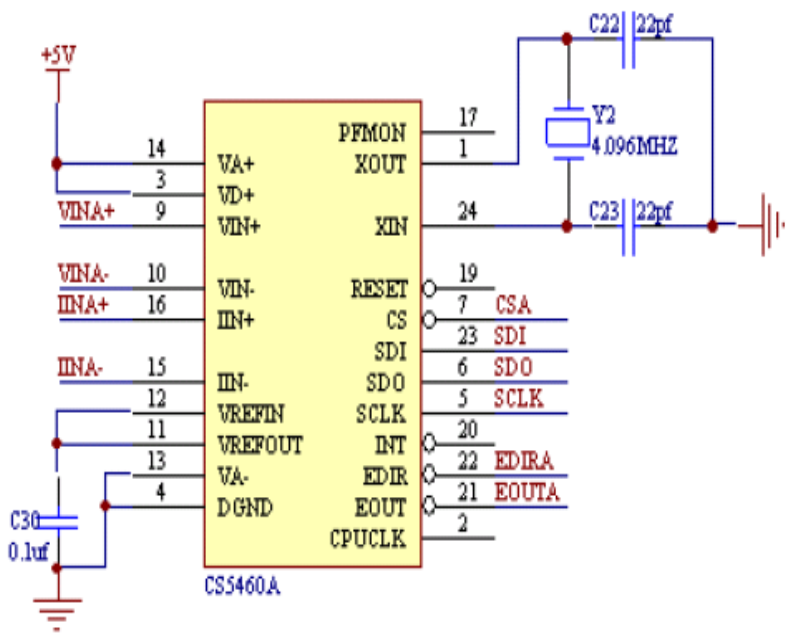

Fig. 3 CS5460A designing of the external circuit

The communication of the slave computer and the host computer in current system adopts the 485-bus method; its principal diagram is as Fig. 4.

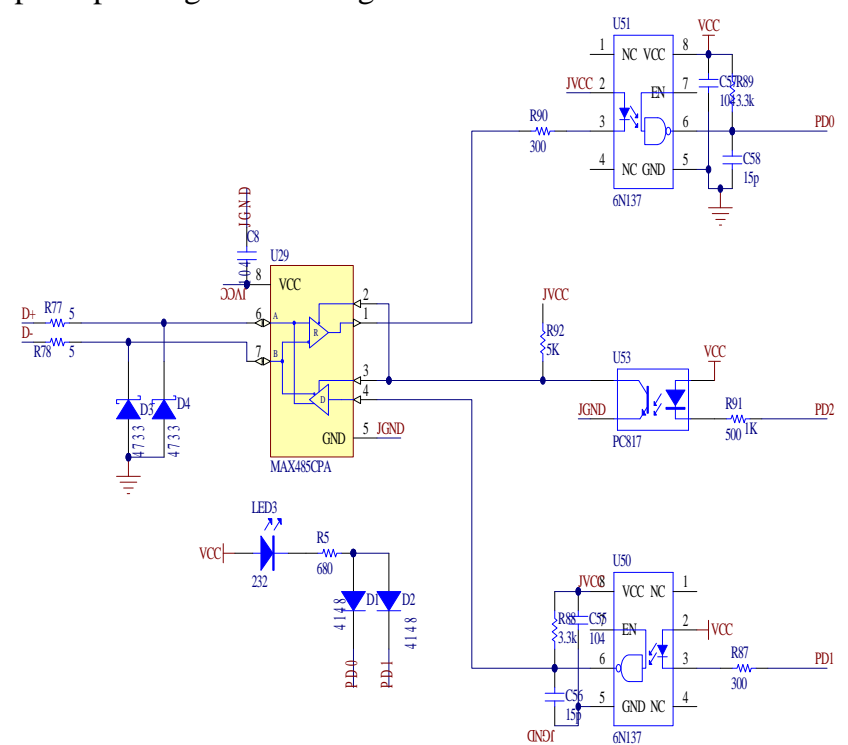

Fig. 4 Isolated 485-bus devises

6 N137 is the photo electricity insulator, PC817 is the low speed photo electricity insulator, and these two apparatuses are used to insulate the bus.

\section{B. Proportion of the Clock and Storage Circuit}

Current system adopts DS3231 to be the systemic clock. This type of chip includes Real-time clock/calendar and static RAM with 31bytes; it communicates with the MCU by means of a simple $\mathrm{I}^{2} \mathrm{C}$ serial interface. The Real-time clock/calendar supplies the function of second, minute, hour, day, week, month and year etc.

The system saves the systemic calibration parameter and the energy value via $24 \mathrm{C} 16$ chip. AT24C16 is a representative chip of AT24C series, which has 2048*8 bits 
capacity and every byte has the endurance of 100000 write/erase cycles.

\section{SOFTWARE DEVISE OF THE SYSTEM}

\section{A. Software Devise of the MCU}

ATMEGA162 transmits data to CS5460A by means of SPI interface, ATMEGA162 reads the virtual value of electricity and voltage of three CS5460A chips orderly and the energy memory, then processes the data numerated [5]. When processing virtual value of current and voltage, the memory just needs to multiply its value by full scale value to get the requisite virtual value of electricity and voltage. The active power is get by the energy translating memory last cycle holding for $200 \mathrm{~ms}$, then add the latest five energy values to get the active power.

Additional, the pulse outputting pins of three CS5460A chips is connected with three external interruption pins of ATMEGA162. When exterior interruption happens, the method of processing is that EDIR pin is judged whether it is high or low level, the direction of energy is negative when it is low level, so a pulse represents energy should be decreased from the general energy; the general energy should be added a pulse represents energy when it is high level.

\section{B. Software devise of the Host Computer}

The host computer software accomplishes the function of Real-time displaying, analyzing, storage and outputting report forms. Current system adopts the Labwindows/CVI to be the virtual instrument exploring platform and makes it as the host computer software [6]. The system functional frame diagram is as Fig. 5 shows. Meanwhile, the upper software provides the function of rest and communication channel changing etc. The main interface of host computer is shown in Fig. 6.

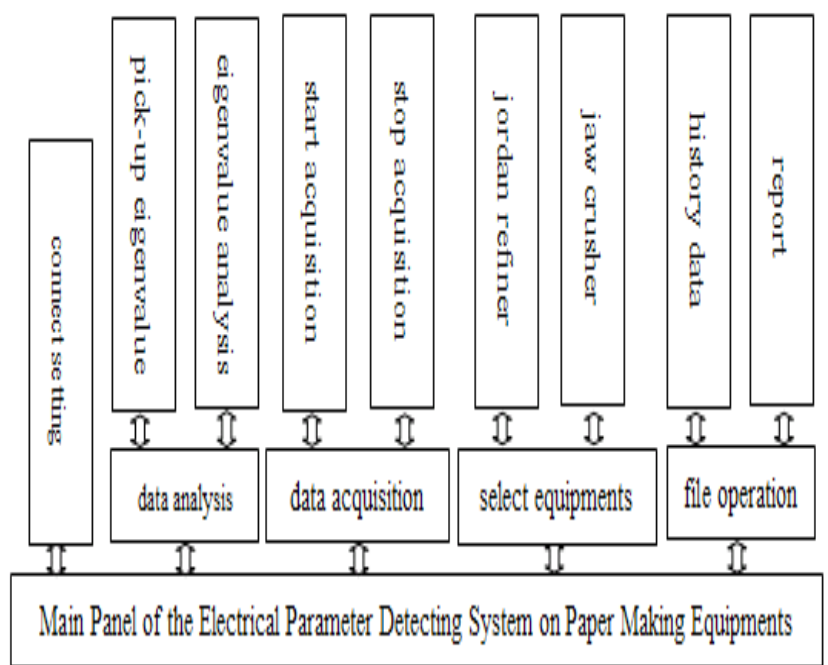

Fig. 5 Host computer software system functional frame diagram

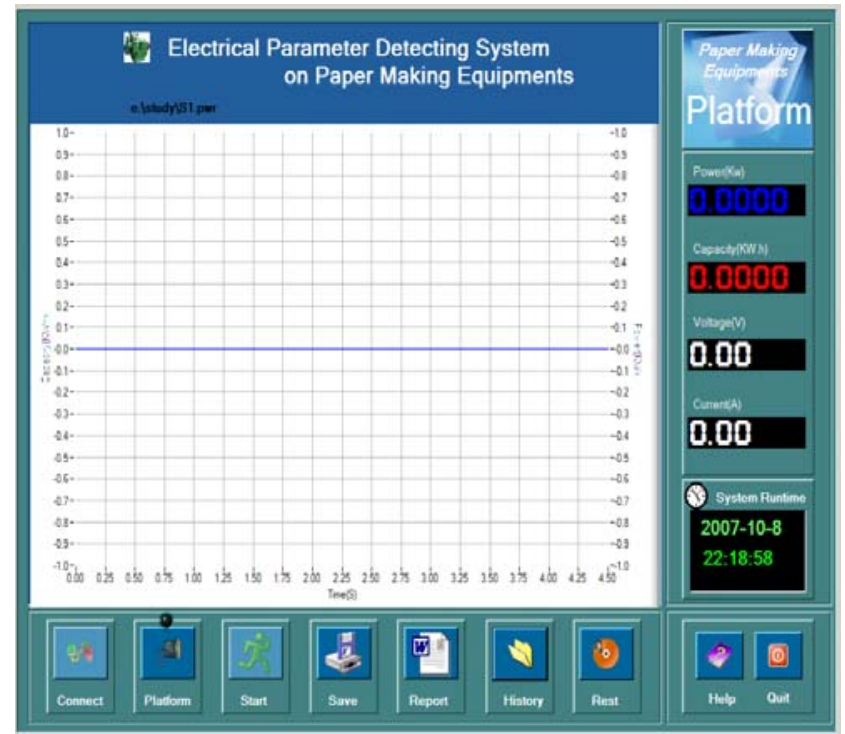

Fig. 6 Main interface of host computer software

\section{CONCLUSIONS}

The electrical parameter detecting system on jordan refinner has been applied in the detecting of three-phase electrical parameter of the main motor of jordan refiner successfully which belongs to Beijing Paper Making Graduate School. Practice has proofed that its measuring precision has achieved 0.1 rates and it provides the characteristic that low cost, simple architecture of circuit, supplying 485 bus interface and high precision.

\section{REFERENCES}

[1] Wang Jianxin, Yang Shifeng, Sui Meili. Labwindows/CVI testing technology and application in project. Beijing, Chemical Industry Press, p163 (2006)

[2] Shang Chunyang, Zhang Gang,Xu Kai. Application of CS5460A in Measure Voltage Current Power. Modern Electronics Technique, (18): 83-86 (2003).

[3] Xiong Shuming.The Design and Implementation of High Accuracy Data Acquisition and Processing System that Based on A CS5460A.Application Research Of Computers, (11): 81-82 (2003).

[4] Johansson Ola M. Refiner control method and system: USP, 6938843 (2005).

[5] [5] Li Hui.The Design of Electric Monitoring System Based on CS5460 and USB Interface. Electrical Measurement \& Instrumentation, (11): 68-71(2006)

[6] Shen wenbin,Guo shiming. Automatic detection system about DK-1 electro-pneumatic brake based on LabWindows/CVI. Foreign Electronic Measurement Technology, (12): 89-92(2006). 